\title{
EXAMPLES OF NONINTEGRABLE ANALYTIC HAMILTONIAN VECTOR FIELDS WITH NO SMALL DIVISORS
}

BY

R. CUSHMAN

\begin{abstract}
Any analytic symplectic diffeomorphism $\Phi$ of a symplectic manifold $M$ is the Poincare map of a real analytic Hamiltonian vector field $X_{H}$. If $\Phi$ does not have an analytic integral, then $X_{H}$ has no analytic integral which is not a power series in $H$. Let $M=\mathbf{R}^{2}$. If $\Phi$ has a finite contact homoclinic point, then $\Phi$ is nonintegrable. Also Moser's polynomial mapping is nonintegrable.
\end{abstract}

Throughout this paper all maps, manifolds and vector fields will be real analytic, unless explicitly stated otherwise.

1. The first theorem states that every symplectic diffeomorphism may be "suspended" to a Hamiltonian vector field. This is an extension of a well known construction [S, p. 797] to Hamiltonian vector fields. In particular every symplectic diffeomorphism is the Poincare mapping of some Hamiltonian vector field, which answers a question in [A, p. 100].

Let $X_{H}$ be a Hamiltonian vector field on a symplectic manifold $(N, \Omega)$ whose flow is $\Phi^{H}: U \subseteq \mathbf{R} \times N \rightarrow N$ where $U$ is an open set in $\mathbf{R} \times N$ containing $\{0\} \times N$. A manifold $M_{h}$ is a cross section of $X_{H}$ in the energy surface $H^{-1}(h)$ if (1) $M_{h}$ is a submanifold of $H^{-1}(h)$ of codimension one such that $\omega=i^{*} \Omega$ is nondegenerate where $i: M_{h} \rightarrow H^{-1}(h)$ is the embedding of $M_{h}$ in $H^{-1}(h)$; (2) for every $m \in M_{h}, X_{H}(m)$ is not tangent to $M_{h}$ at $m$; and (3) if $p \in H^{-1}(h)$ then there is $t=t(p) \in \mathbf{R} \backslash\{0\}$ such that $(t, p) \in U$ and $\Phi^{H}(t, p) \in M_{h}$. For each $p \in H^{-1}(h)$ let $T(p)=\{\inf |t| \mid t \neq 0$ and $\left.\Phi^{H}(t, p) \in M_{h}\right\}$. Then the diffeomorphism $\Phi: M_{h} \rightarrow M_{h}: m \rightarrow \Phi^{H}(T(m), m)$ is the Poincare map of $X_{H}$ corresponding to the cross section $M_{h}$. It is well known [AM, p. 178] that $\Phi$ is a symplectic diffeomorphism of $\left(M_{h}, \omega\right)$.

THEOREM 1.1. Let $\Phi: M \rightarrow M$ be a symplectic diffeomorphism of the connected symplectic manifold $(M, \omega)$. Then there is a connected symplectic manifold $(N, \Omega)$ and a Hamiltonian vector field $X_{H}$ on $N$ such that in every energy

Received by the editors June 7, 1976.

AMS (MOS) subject classifications (1970). Primary 58F05; Secondary $70 \mathrm{H} 99$.

Key words and phrases. Symplectic diffeomorphism, suspension, integrable, Moser's diffeomorphism, homoclinic point.

๑ American Mathematical Society 1978 
surface $H^{-1}(s), X_{H}$ has

(1) a cross section $M_{s}$ diffeomorphic to $M$ by $j_{s}$ and

(2) a Poincare map $\Phi_{1}^{H}: M_{s} \rightarrow M_{s}$, where $\Phi^{H}$ is the flow of $X_{H}$ such that $\Phi=j_{s} \circ \Phi_{1}^{H} \circ j_{s}^{-1}$.

Proof. On the symplectic manifold $M \times \mathbf{R}^{2}$ with symplectic form $\tilde{\Omega}=\omega$ $+d t \wedge d s$ define an action of the integers by

$$
\Xi: \mathbf{Z} \times\left(M \times \mathbf{R}^{2}\right) \rightarrow M \times \mathbf{R}^{2}:(n,(m, t, s)) \rightarrow\left(\Phi^{n}(m), t-n, s\right) .
$$

Say that two points $(m, t, s)$ and $\left(m^{\prime}, t^{\prime}, s^{\prime}\right)$ of $M \times \mathbf{R}^{2}$ are equivalent if and only if they lie in the same orbit of the action $\Xi$, that is, if and only if for some $n \in \mathbf{Z},\left(m^{\prime}, t^{\prime}, s^{\prime}\right)=\Xi(n,(m, t, s))$. Let $N$ be the set of all orbits of the action $\Xi$ and let $\pi: M \times \mathbf{R}^{2} \rightarrow N$ be the map which assigns to $(m, t, s)$ the orbit $\left\{\Xi(n,(m, t, s)) \in M \times \mathbf{R}^{2} \mid n \in \mathbf{Z}\right\}$. Then $N$ is a manifold and $\pi$ is a diffeomorphism because $\Xi$ is a free and properly discontinuous action $[K N, p$. 44]. Also $N$ is connected because $\pi$ is continuous and $M \times \mathrm{R}^{2}$ is connected. Since

$$
\Xi_{n}^{*} \tilde{\Omega}=\left(\Phi^{n}\right)^{*} \omega+d(t-n) \wedge d s=\omega+d t \wedge d s=\tilde{\Omega},
$$

the two form $\tilde{\Omega}$ on $M \times \mathbf{R}^{2}$ induces a two form $\Omega$ on $N$ such that $\tilde{\Omega}=\pi^{*} \Omega$. Because $\pi$ is a local diffeomorphism and $\tilde{\Omega}$ is closed and nondegenerate, $\Omega$ is closed and nondegenerate. Hence $(N, \Omega)$ is a symplectic manifold.

Let $T$ be the vector field on $M \times \mathbf{R}^{2}$ whose flow is

$$
\tilde{\Psi}: \mathbf{R} \times\left(M \times \mathbf{R}^{2}\right) \rightarrow M \times \mathbf{R}^{2}:(u,(m, t, s)) \rightarrow(m, t+u, s) ;
$$

then because $i_{T} \tilde{\Omega}=d s, T$ is the Hamiltonian vector field corresponding to the Hamiltonian function $\tilde{H}: M \times \mathbf{R}^{2} \rightarrow \mathbf{R}:(m, t, s) \rightarrow s$, that is, $X_{\tilde{H}}=T$. Because

$$
\begin{aligned}
\Xi_{n} \circ \tilde{\Psi}_{u}(m, t, s) & =\Xi_{n}(m, t+u, s)=\left(\Phi^{n}(m), t+u-n, s\right) \\
& =\tilde{\Psi}_{u}\left(\Phi^{n}(m), t-n, s\right)=\tilde{\Psi}_{u} \circ \Xi_{n}(m, t, s),
\end{aligned}
$$

$\tilde{\Psi}$ induces a flow $\Psi$ on $N$ which gives rise to a vector field $X$ on $N$ such that $\pi^{*} X=X_{\tilde{H}} . \tilde{H}$ induces a function $H: N \rightarrow \mathbf{R}$ such that $\tilde{H}=\pi^{*} H$ because

$$
\begin{aligned}
\left(\Xi_{n}^{*} \tilde{H}\right)(m, t, s) & =\tilde{H}\left(\Xi_{n}(m, t, s)\right)=\tilde{H}\left(\Phi^{n}(m), t-n, s\right) \\
& =s=\tilde{H}(m, t, s) .
\end{aligned}
$$

Now $X=X_{H}$, the Hamiltonian vector field corresponding to the Hamiltonian function $H: N \rightarrow R$, since

$$
\pi^{*} d H=d \tilde{H}=i_{X_{\dot{H}}} \tilde{\Omega}=i_{\pi^{*} X}\left(\pi^{*} \Omega\right)=\pi^{*}\left(i_{X} \Omega\right)
$$

implies $d H=i_{X} \Omega$ because $\pi$ is a local diffeomorphism.

Fix $s \in \mathbf{R}$. Because $\tilde{M}_{s}=M \times\{(0, s)\}$ is a cross section of $X_{\dot{H}}$ in $\tilde{H}^{-1}(s)$, 
$M_{s}=\pi\left(\tilde{M}_{s}\right)$ is a cross section of $X_{H}$ in $H^{-1}(s)$. Note that $\pi \mid \tilde{M}_{s}$ is injective for

$$
\begin{aligned}
\left(\pi \mid \tilde{M}_{s}\right)^{-1}(\pi(m, 0, s)) & =\pi^{-1}(\pi(m, 0, s)) \cap \tilde{M}_{s} \\
& =\left\{\left(\Phi^{n}(m),-n, s\right) \in M \times \mathbf{R}^{2} \mid n \in \mathbf{Z}\right\} \cap \tilde{M}_{s} \\
& =(m, 0, s),
\end{aligned}
$$

which implies that $\pi \mid \tilde{M}_{s}: \tilde{M}_{s} \rightarrow M$ is a diffeomorphism. The map $i_{s}: M \rightarrow \tilde{M}_{s}$ : $m \rightarrow(m, 0, s)$ being a diffeomorphism implies that the map $j_{s}: M \rightarrow M_{s}$ : $m \rightarrow \pi\left(i_{s}(m)\right)$ is a diffeomorphism. From

$$
\begin{aligned}
\tilde{\Psi}_{1}\left(\Xi_{n}(m, 0, s)\right) & =\tilde{\Psi}_{1}\left(\Phi^{n}(m),-n, s\right) \\
& =\left(\Phi^{n-1}(\Phi(m)),-(n-1), s\right) \\
& =\Xi_{n-1}(\Phi(m), 0, s)
\end{aligned}
$$

we obtain $\Psi_{1} \pi(m, 0, s)=\pi(\Phi(m), 0, s)$ which gives (1) $\Psi_{1}: M_{s} \rightarrow M_{s}$ and $\Psi_{1}$ is the Poincare map of $X_{H}$ in $H^{-1}(s)$ with respect to the cross section $M_{s}$ and (2) $\Phi=j_{s}^{-1} \Psi_{1} j_{s}$. This proves the theorem.

The Hamiltonian vector field $X_{H}$ constructed in the above theorem is the Hamiltonian suspension of the symplectic diffeomorphism $\Phi$.

Quite unusual Hamiltonian vector fields can be constructed using the Hamiltonian suspension theorem. For example on $\left(\mathbf{R}^{2}, d x \wedge d y\right)$ the Hamiltonian suspension of the symplectic diffeomorphism

$$
R_{\theta}: \mathbf{R}^{2} \rightarrow \mathbf{R}^{2}:\left(\begin{array}{l}
x \\
y
\end{array}\right) \rightarrow\left(\begin{array}{cc}
\cos \theta & -\sin \theta \\
\sin \theta & \cos \theta
\end{array}\right)\left(\begin{array}{l}
x \\
y
\end{array}\right)
$$

where $\theta / 2 \pi$ is irrational, has exactly one periodic orbit in every energy surface $H^{-1}(s)$ (which is compact) because ( $\left.{ }_{0}^{0}\right)$ is the only fixed point of $R_{\theta}^{n}$ and fixed points of some iterate of $R_{\theta}$ correspond to periodic orbits of $X_{H}$ and conversely.

The rest of this paper applies the Hamiltonian suspension theorem to constructing nonintegrable Hamiltonian vector fields. A function $G: N \rightarrow \mathbf{R}$ is an integral of the Hamiltonian vector field $X_{H}$ on the connected symplectic manifold $(N, \Omega)$ if and only if the Lie derivative of $G$ with respect to $X_{H}$ is identically zero. The Hamiltonian vector field $X_{H}$ on $(N, \Omega)$ is nonintegrable if every integral is of the form $H^{*} g: N \rightarrow \mathbf{R}: n \rightarrow g(H(n))$ for some function $g$ : $\mathbf{R} \rightarrow \mathbf{R} . X_{H}$ is integrable if there is an integral which is not equal to $H^{*} g$ for any $g: \mathbf{R} \rightarrow \mathbf{R}$. We also define the concepts of integral, integrable and nonintegrable for symplectic diffeomorphisms. A function $F: M \rightarrow \mathbf{R}$ is an integral of the symplectic diffeomorphism $\Phi: M \rightarrow M$ of the connected symplectic manifold $(M, \omega)$ if for every $m \in M, F(\Phi(m))=F(m) . \Phi$ is nonintegrable if it has no constant integral, while $\Phi$ is integrable if it has a 
nonconstant integral. Recall that in the above definitions all maps, manifolds and vector fields are real analytic.

The next theorem reduces the problem of constructing nonintegrable Hamiltonian vector fields to constructing nonintegrable symplectic diffeomorphisms.

THEOREM 1.2. If $\Phi: M \rightarrow M$ is a nonintegrable symplectic diffeomorphism of a connected symplectic manifold $(M, \omega)$, then the Hamiltonian suspension of $\Phi$, $X_{H}$, is a nonintegrable Hamiltonian vector field on the connected symplectic manifold $(N, \Omega)$.

Proof. The same notation as in the proof of Theorem 1.1 will be used. Suppose that $J: N \rightarrow \mathbf{R}$, then for all $(m, t, s) \in M \times \mathbf{R}^{2}$

$$
\begin{aligned}
\left(\pi^{*} J\right)\left(\Phi^{n}(m), t, s\right) & =\left(\pi^{*} J\right)\left(\Xi_{n}(m, t, s)\right) \\
& =J\left(\pi\left(\Xi_{n}(m, t, s)\right)\right)=J(\pi(m, t, s)) \\
& =\left(\pi^{*} J\right)(m, t, s) .
\end{aligned}
$$

Furthermore suppose that $J: N \rightarrow \mathbf{R}$ is an integral of $X_{H}$, then for all $u \in \mathbf{R}$,

$$
\begin{aligned}
\pi^{*} J(m, t+u, s) & =\pi^{*} J\left(\tilde{\Psi}_{u}(m, t, s)\right) \quad \text { where } \tilde{\Psi}_{u} \text { is the flow of } T \\
& =J\left(\pi \tilde{\Psi}_{u}(m, t, s)\right)=J\left(\Psi_{u} \pi(m, t, s)\right)
\end{aligned}
$$
by definition of the flow $\Psi$ of $X_{H}$

$$
\begin{aligned}
& =J(\pi(m, t, s)) \quad \text { since } J \text { is an integral of } X_{H} \\
& =\pi^{*} J(m, t, s),
\end{aligned}
$$

that is, $\pi^{*} J: M \times \mathbf{R}^{2} \rightarrow \mathbf{R}$ is an integral of $X_{\tilde{H}}=T$. From (*) and (**) we obtain

$$
\pi^{*} J\left(\Phi^{n}(m), t, s\right)=\pi^{*} J(m, t+n, s)=\pi^{*} J(m, t, s),
$$

that is, for all $(t, s) \in \mathbf{R}^{2}$ the map $\pi^{*} J(\cdot, t, s): M \rightarrow \mathbf{R}: m \rightarrow \pi^{*} J(m, t, s)$ is an integral of the diffeomorphism $\Phi: M \rightarrow M$ which is constant by hypothesis. From (**) we see that the map $\mathcal{T}: \mathbf{R} \rightarrow \mathbf{R}: s \rightarrow \pi^{*} J(\cdot, \cdot, s)$ is well defined. Since $\tilde{H}: M \times \mathbf{R}^{2} \rightarrow \mathbf{R}:(m, t, s) \rightarrow s$, we have

$$
\tilde{H}^{*} \mathcal{T}(m, t, s)=\mathscr{T} \tilde{H}(m, t, s)=\mathcal{T}(s)=\pi^{*} J(m, t, s) .
$$

Because $\tilde{H}=H \circ \pi, \pi^{*} J=(H \circ \pi)^{*} \mathfrak{T}=\pi^{*}\left(H^{*} \mathfrak{T}\right)$ which implies $J=H^{*} \mathfrak{T}$ since $\pi$ is a local diffeomorphism. Therefore $X_{H}$ is nonintegrable.

2. Here we show that the polynomial mapping

$$
M: \mathbf{R}^{2} \rightarrow \mathbf{R}^{2}:\left(\begin{array}{l}
x \\
y
\end{array}\right) \rightarrow\left[\begin{array}{l}
\left(x+y^{3}\right) \cos \theta-y \sin \theta \\
\left(x+y^{3}\right) \sin \theta+y \cos \theta
\end{array}\right]
$$


where $\theta / 2 \pi$ is irrational is a nonintegrable symplectic diffeomorphism of $\left(\mathbf{R}^{2}\right.$, $d x \wedge d y)$.

The mapping $M$ has the following properties: (1) for every positive integer $n, M^{2 n}$ has at most $3^{2 n}$ fixed points; (2) in every open neighbourhood $U$ of 0 there is a positive integer $p$ and a $w \in U \backslash\{0\}$ such that $M^{2 p_{w}}=w$. Property (1) is proved by the following argument which is taken from [SM, pp. 246-247]. The map

$$
M^{-1}: \mathbf{R}^{2} \rightarrow \mathbf{R}^{2}:\left(\begin{array}{l}
x \\
y
\end{array}\right) \rightarrow\left(\begin{array}{c}
x \cos \theta+y \sin \theta+(x \sin \theta-y \cos \theta)^{3} \\
-x \sin \theta+y \cos \theta
\end{array}\right)
$$

is also a polynomial mapping. The point $\left(\begin{array}{l}x \\ y\end{array}\right)$ is a fixed point of $M^{2 n}$ if and only if $\left(\begin{array}{l}x_{-}-n \\ y_{n}\end{array}\right)=M^{-n}\left(\begin{array}{l}x \\ y\end{array}\right)=M^{n}\left(\begin{array}{l}x \\ y\end{array}\right)=\left(\begin{array}{l}x_{n} \\ y_{n}\end{array}\right)$ which is equivalent to the polynomial equations

$$
\begin{aligned}
& x_{-n}-x_{n}=0, \\
& y_{-n}-y_{n}=0 .
\end{aligned}
$$

Introducing complex conjugate "coordinates" $z=x+i y, \bar{z}=x-i y$ on $\mathbf{R}^{2}$, the map $M$ becomes $z_{1}=M(z, \bar{z})=\lambda\left(z+(i / 8)(z-\bar{z})^{3}\right)$ and $M^{-1}$ becomes $z_{-1}=M^{-1}(z, \bar{z})=\bar{\lambda} z+\frac{1}{8}(i \bar{\lambda} z+\lambda \bar{z})^{3}$ where $\lambda=e^{i \theta}$. A straightforward induction argument shows that the terms of highest degree of the polynomials $x_{-n}-x_{n}$ and $y_{-n}-y_{n}$ are respectively

$$
X=a_{n}\left[\cos \theta y^{3^{n}}-(x \sin \theta-y \cos \theta)^{3^{n}}\right] \text { and } Y=a_{n} \sin \theta y^{3^{n}}
$$

where $a_{n}=(\sin \theta)^{3+3^{2}+\cdots+3^{n-1}}$. Since $a_{n} \neq 0$ for all $n$ and $\sin \theta \neq 0, X$ and $Y$ are relatively prime polynomials which implies that $x_{-n}-y_{n}$ and $y_{-n}-y_{n}$ ave relatively prime. Hence by Bezout's theorem $x_{-n}-x_{n}$ and $y_{-n}-y_{n}$ have at most $3^{2 n}$ common zeroes which proves property (1). To prove property (2) we observe that the symplectic diffeomorphism $S$ of an open neighbourhood $V$ of 0 of $\mathbf{R}^{2}$ whose generating function is

$$
G(x, \eta)=x \eta-\frac{1}{16}\left(x^{3}+x \eta^{2}\right)+\frac{1}{64}\left(x^{4}-6 x^{2} \eta^{2}+\eta^{4}\right)
$$

(recall that $\xi=G_{x}, y=G_{\eta}$ implicitly defines $S$ ) conjugates $M$ into Birkhoff normal form up to terms of order three, that is

$$
S M S^{-1}: V \subseteq \mathbf{R}^{2} \rightarrow \mathbf{R}^{2}:\left(\begin{array}{l}
x \\
y
\end{array}\right) \rightarrow\left(\begin{array}{cc}
\cos \Phi & -\sin \Phi \\
\sin \Phi & \cos \Phi
\end{array}\right)\left(\begin{array}{l}
x \\
y
\end{array}\right)+\left(\begin{array}{l}
R_{1}(x, y) \\
R_{2}(x, y)
\end{array}\right)
$$

where $\Phi(x, y)=\theta+\beta\left(x^{2}+y^{2}\right), \beta=-\frac{3}{8}$ and $R_{1}, R_{2}$ are real analytic functions beginning with terms of order four. Since $\beta \neq 0$ we may apply the Birkhoff fixed point theorem [SM, pp. 174-179] to obtain property (2).

We now suppose that $H(x, y)=H_{m}(x, y)+\ldots$ is a nonconstant real analytic integral of $M$ which begins with $m$ th order terms $m \geqslant 1$. On comparing $m$ th order terms of $H(M(x, y))=H(x, y)$ we obtain 


$$
H_{m}(x, y)=H_{m}\left(R_{\theta}(x, y)\right)
$$

where

$$
R_{\theta}\left(\begin{array}{l}
x \\
y
\end{array}\right)=\left(\begin{array}{cc}
\cos \theta & -\sin \theta \\
\sin \theta & \cos \theta
\end{array}\right)\left(\begin{array}{l}
x \\
y
\end{array}\right)
$$

and $\theta / 2 \pi$ is irrational. Introducing complex conjugate co-ordinates $z, \bar{z}$ for $\mathbf{R}^{2}$ the real homogeneous polynomial $H_{m}$ of degree $m$ becomes

$$
Q_{m}(z, \bar{z})=\sum_{\mu+\nu=m} q_{\mu \nu} z^{\mu \bar{z}^{\nu}}
$$

where $q_{\mu \nu}=\bar{q}_{\nu \mu}$. The map $R_{\theta}$ becomes $R^{\lambda} z=\lambda z, \lambda=e^{i \theta}$. Therefore (*) implies $Q_{m}(z, \bar{z})=Q_{m}(\lambda z, \overline{\lambda z})$ which upon comparing coefficients gives

$$
q_{\mu \nu}=\lambda^{\mu} \bar{\lambda}^{v} q_{\mu \nu}=\lambda^{\mu-\nu} q_{\mu \nu} \text {. }
$$

Since $H_{m} \neq 0, Q_{m} \neq 0$ and therefore for some $\mu_{0}, \nu_{0}, q_{\mu_{0} \nu_{0}} \neq 0$. Thus $1=$ $\lambda^{\mu_{0}-\nu_{0}}=e^{i\left(\mu_{0}-\nu_{0}\right) \theta}$ by (**). Since $\theta / 2 \pi$ is irrational $\left(\mu_{0}-\nu_{0}\right)(\theta / 2 \pi)$ is an integer if and only if $\mu_{0}=\nu_{0}$. Thus $m=\mu_{0}+\nu_{0}=2 \nu_{0} \geqslant 2$ and

$$
Q_{m}(z, \bar{z})=q(z \bar{z})^{m / 2}=q\left(x^{2}+y^{2}\right)^{m / 2}=H_{m}(x, y)
$$

where $q=q_{\mu_{0} \nu_{0}} \neq 0$.

Upon dividing $H$ by $q$ we may assume that the integral of $M$ is of the form $H(x, y)=\left(x^{2}+y^{2}\right)^{m / 2}+H_{m+1}(x, y)$ where $H_{m+1}$ is a real analytic function beginning with terms of order at least $m+1$.

Modifying an argument in [M1, pp. 416-417], we construct a differentiable diffeomorphism

$$
\left.P: A_{\hat{\boldsymbol{\rho}}}=\right] 0, \hat{\rho}\left[\times\left[0,2 \pi\left[\rightarrow \mathbf{R}^{2} \backslash\{0\}\right.\right.\right.
$$

such that $\hat{M}=P \circ M \circ P^{-1}: A_{\hat{\rho}} \rightarrow A_{\hat{\rho}}:\left(\begin{array}{l}h \\ \psi\end{array}\right) \rightarrow\left(\begin{array}{c}h \\ \psi+\alpha(h)\end{array}\right)$. First the mapping

$$
P_{1}: A_{\infty} \rightarrow \mathbf{R}^{2} \backslash\{0\}:\left(\begin{array}{l}
R \\
\vartheta
\end{array}\right) \rightarrow\left(\begin{array}{l}
x \\
y
\end{array}\right)=\left(\begin{array}{ll}
R^{1 / m} & \cos \vartheta \\
R^{1 / m} & \sin \vartheta
\end{array}\right)
$$

is a differentiable diffeomorphism with

$$
P_{1}^{*}(d x \wedge d y)=\frac{1}{2} d R^{2 / m} \wedge d \vartheta .
$$

Second, we show that for $\rho \in] 0,1[$ sufficiently small the mapping

$$
P_{2}: A_{\rho} \rightarrow A_{\hat{\rho}}:\left(\begin{array}{l}
R \\
\vartheta
\end{array}\right) \rightarrow\left(\begin{array}{l}
h \\
\vartheta
\end{array}\right)=\left(\begin{array}{c}
H\left(P_{1}(R, \vartheta)\right) \\
\vartheta
\end{array}\right)
$$

is a diffeomorphism.

Since det $d P_{2}=\partial h / \partial R=1+\partial H_{m+1}\left(P_{1}(R, \vartheta)\right) / \partial R$ and 
$\sup _{(R, \vartheta) \in A_{\rho}}\left|\frac{\partial H_{m+1}}{\partial R}\left(P_{1}(R, \vartheta)\right)\right| \leqslant \frac{1}{m} \rho^{1 / m-1} \sup _{(R, \vartheta) \in A_{\rho}}|f(R, \vartheta)|$ where $f(R, \vartheta)=\cos \vartheta \frac{\partial H_{m+1}}{\partial x}\left(R^{1 / m} \cos \vartheta, R^{1 / m} \sin \vartheta\right)$ $+\sin \vartheta \frac{\partial H_{m+1}}{\partial y}\left(R^{1 / m} \cos \vartheta, R^{1 / m} \sin \vartheta\right)$

$<\frac{1}{m} \rho^{1 / m-1}\left(\rho^{1 / m}\right)^{m} c$ since $\partial H_{m+1} / \partial x, \partial H_{m+1} / \partial y$ are real analytic and begin with terms of order at least $m$.

Also $c=\sup _{\vartheta \in[0,2 \pi]}|f(1, \vartheta)|$ does not depend on $\rho$ or $\vartheta$.

$=\frac{1}{m} \rho^{1 / m} c$,

for some $\rho \in] 0,1[$,

$$
\frac{\partial h}{\partial R}(R, \vartheta)>\frac{1}{2}
$$

for all $(R, \vartheta) \in A_{\rho}$ and $\operatorname{det} d P_{2} \neq 0$ for all $(R, \vartheta) \in A_{\rho}$. Thus $P_{2}$ is a local diffeomorphism. Because $\lim _{R \rightarrow 0^{+}} h(R, \vartheta)=0, \lim _{R \rightarrow 0^{+}} \partial h(R, \vartheta) / \partial R=1$ and (**), there is $\hat{\rho} \in] 0,1[$ such that for all $\vartheta \in[0,2 \pi[$ the function

$$
\left.h_{\vartheta}:\right] 0, \rho[\rightarrow] 0, \hat{\rho}[: R \rightarrow h(R, \vartheta)
$$

is a diffeomorphism. Thus $P_{2}$ is one-to-one and hence is a diffeomorphism. Also $\left(P_{2}^{-1}\right)^{*}\left(\frac{1}{2} d R^{2 / m} \wedge d \vartheta\right)=p(h, \vartheta) d h \wedge d \vartheta$ where $p(h, \vartheta)=$ $\left(P_{2}^{-1}\right)^{*} q(h, \vartheta)$ and

$$
q(R, \vartheta)=\frac{1}{R^{1-2 / m} \partial h(R, \vartheta) / \partial R}
$$

Since $q(R, \vartheta)>0$ for $(R, \vartheta) \in A_{\rho}$ by $(* *), p(h, \vartheta)>0$ for $(h, \vartheta) \in A_{\hat{\rho}}$. Put $\tilde{P}=P_{2} \circ P_{1}^{-1}$, then

$$
\tilde{M}=\tilde{P} \circ M \circ \tilde{P}^{-1}: A_{\hat{\rho}} \rightarrow A_{\hat{\rho}}:\left(\begin{array}{l}
h \\
\vartheta
\end{array}\right) \rightarrow\left(\begin{array}{l}
h_{1} \\
\vartheta_{1}
\end{array}\right)=\left(\begin{array}{l}
h \\
\vartheta_{1}
\end{array}\right)
$$

because $H$ is an integral for $M$. Also, by the way $\tilde{P}$ was constructed and because $M$ is symplectic

$$
(\tilde{M})^{*}(p(h, \vartheta)) d h \wedge d \vartheta=p(h, \vartheta) d h \wedge d \vartheta .
$$

Integrating (+) over the set $S$ :

$$
\begin{gathered}
0<h_{0} \leqslant h \leqslant h_{0}+\eta<\hat{\rho}, \\
0 \leqslant \vartheta^{\prime}<\vartheta \leqslant \vartheta^{\prime \prime}<2 \pi
\end{gathered}
$$

gives 


$$
\int_{\tilde{M} S} p(h, \vartheta) d h \wedge d \vartheta=\int_{\tilde{M} S} \tilde{M}^{*}(p(h, \vartheta) d h \wedge d \vartheta)=\int_{S} p(h, \vartheta) d h \wedge d \vartheta
$$

that is,

$$
\frac{1}{\eta} \int_{h_{0}}^{h_{0}+\eta}\left(\int_{\vartheta^{\prime}}^{\vartheta^{\prime \prime}} p\left(h_{0}+\eta, \vartheta\right) d \vartheta\right) d h=\frac{1}{\eta} \int_{h_{0}}^{h_{0}+\eta}\left(\int_{\vartheta_{\mathrm{i}}^{\prime}}^{\vartheta_{i}^{\prime \prime}} p\left(h_{0}+\eta, \vartheta\right) d \vartheta\right) d h
$$

which letting $\eta \rightarrow 0$ gives

$(* * *) \quad \int_{\vartheta^{\prime}}^{\vartheta^{\prime \prime}} p\left(h_{0}, \vartheta\right) d \vartheta=\int_{\vartheta_{1}^{\prime}}^{\vartheta_{\prime}^{\prime \prime}} p\left(h_{0}, \vartheta\right) d \vartheta \quad$ for all $\left.h_{0} \in\right] 0, \hat{\rho}[$.

Finally, define the mapping $P_{3}: A_{\hat{\rho}} \rightarrow A_{\hat{\rho}}:\left(\begin{array}{c}h \\ \vartheta\end{array}\right) \rightarrow\left(\begin{array}{c}h \\ \Psi\end{array}\right)$ where $\Psi(h, \vartheta)=$ $2 \pi \int_{0}^{\vartheta} p(h, \vartheta) d \vartheta / \int_{0}^{2 \pi} p(h, \vartheta) d \vartheta$.

Since $p(h, \vartheta)>0$ for all $(h, \vartheta) \in A_{\hat{\rho}}, \Psi$ is defined and maps [0,2 2 [ into itself. $P_{3}$ is a local diffeomorphism of $A_{\hat{\rho}}$ into itself since det $d P_{3}=\partial \Psi / \partial \vartheta=$ $2 \pi p(h, \vartheta)>0$. Since

$$
\Psi_{h}:[0,2 \pi[\rightarrow[0,2 \pi[: \vartheta \rightarrow \Psi(h, \vartheta)
$$

is a diffeomorphism for every $h \in] 0, \hat{\rho}\left[, P_{3}\right.$ is injective and hence a diffeomorphism.

Let $P=P_{3} \circ \tilde{P}=P_{3} \circ P_{2} \circ P_{1}^{-1}$, then

$$
\hat{M}=P \circ M \circ P^{-1}: A_{\hat{\rho}} \rightarrow A_{\hat{\rho}}:\left(\begin{array}{c}
h \\
\Psi
\end{array}\right) \rightarrow\left(\begin{array}{c}
h_{1} \\
\Psi_{1}
\end{array}\right)=\left(\begin{array}{c}
h \\
\Psi_{1}
\end{array}\right) .
$$

From (***) we obtain

$$
\Psi\left(h_{0}, \vartheta^{\prime \prime}\right)-\Psi\left(h_{0}, \vartheta^{\prime}\right)=\Psi_{1}\left(h_{0}, \vartheta_{1}^{\prime \prime}\right)-\Psi_{1}\left(h_{0}, \vartheta_{1}^{\prime}\right)
$$

which implies that the function $\vartheta \rightarrow \Psi_{1}\left(h_{0}, \vartheta_{1}\right)-\Psi\left(h_{0}, \vartheta\right)$ does not depend on $\vartheta$ and hence is a function $\alpha$ of $h_{0}$ alone. Consequently

$$
\left(\begin{array}{l}
h_{1} \\
\Psi_{1}
\end{array}\right)=\hat{M}\left(\begin{array}{c}
h \\
\Psi
\end{array}\right)=\left(\begin{array}{c}
h \\
\Psi+\alpha(h)
\end{array}\right) \text {. }
$$

For some positive integer $p, M^{2 p}$ has a fixed point $\left(\begin{array}{l}x_{0} \\ y_{0}\end{array}\right)$ in $P^{-1}\left(A_{\hat{\rho}}\right)$. Therefore $P\left(\begin{array}{l}x_{0} \\ y_{0}\end{array}\right)=\left(\begin{array}{l}h_{0} \\ \Psi_{0}\end{array}\right)$ is a fixed point of $\hat{M}^{2 p}$ in $A_{\hat{\rho}}$. But $\hat{M}^{2 p}\left(\begin{array}{l}h \\ \Psi\end{array}\right)=\left(\begin{array}{c}h \\ \Psi+2 p \alpha(h)\end{array}\right)$ $=\left(\begin{array}{l}h_{2 p} \\ \Psi_{2 p}\end{array}\right)$ so $2 p \alpha\left(h_{0}\right)=2 m \pi$ for some positive integer $m$. Thus $\hat{M}$ leaves the curve $h=h_{0}$ fixed which implies $M$ leaves the curve $P^{-1}\left\{\left(\begin{array}{l}h \\ \Psi\end{array}\right) \in A_{\hat{\rho}} \mid h=h_{0}\right\}$ which is impossible, since $M^{2 q}$ has only finitely many fixed points. Therefore the assumption that $M$ has a nonconstant analytic integral is false; that is, $M$ is nonintegrable.

In [B] it is shown that the set $\mathscr{N}$ of real analytic symplectic diffeomorphisms of $\left(\mathbf{R}^{2}, d x \wedge d y\right)$ with the following properties is generic.

$M \in \mathfrak{N}$ if and only if (1) $M(0)=0$,

$$
d M(0)=\left(\begin{array}{cc}
\cos \theta & -\sin \theta \\
\sin \theta & \cos \theta
\end{array}\right), \text { where } \theta / 2 \pi \text { is irrational, }
$$


and (3) there is a sequence of positive integers $m_{n} \rightarrow \infty$ and points $x_{n} \rightarrow 0$ such that $x_{n}$ is an isolated fixed point of $M^{m_{n}}$, that is, there is an open neighbourhood $U_{n}$ of $x_{n}$ such that $M^{m_{n}}$ has no fixed points in $U_{n} \backslash\left\{x_{n}\right\}$. Except in the last paragraph where property (3) is used to obtain the contradiction, the above argument shows that every $M \in \mathfrak{N}$ is nonintegrable.

3. Here we show that if $M$ is a symplectic diffeomorphism of $\left(\mathbf{R}^{2}, d x \wedge d y\right)$ with a finite contact homoclinic point, then $M$ is nonintegrable.

The point $0 \in \mathbf{R}^{2}$ is a hyperbolic fixed point of $M$ if $M(0)=0$ and $d M(0)$ has no eigenvalue of modulus 1 . Since $M$ is symplectic, $d M(0)$ is conjugate by an element of $\operatorname{Sl}(2, \mathbf{R})$ to $\left(\begin{array}{ll}\lambda & 0 \\ 0 & 1 / \lambda\end{array}\right)$ where $|\lambda|>1$. Without loss of generality we may assume that $d M(0)=\left(\begin{array}{cc}\lambda & 0 \\ 0 & 1 / \lambda\end{array}\right)$ with $|\lambda|>1$.

The stable manifold of $M$ at 0 is the set $W_{M}^{s}(0)=\left\{m \in \mathbf{R}^{2} \mid \lim _{n \rightarrow \infty} M^{n} m=\right.$ $0\}$ which is clearly $M$ invariant, that is $M\left(W_{M}^{s}(0)\right) \subseteq W_{M}^{s}(0)$.

Similarly the unstable manifold of $M$ at 0 is the set $W_{M}^{u}(0)=\{m \in$ $\left.\mathbf{R}^{2} \mid \lim _{n \rightarrow \infty} M^{-n} m=0\right\}$ which is also $M$ invariant. By the stable manifold theorem [S, p. 751] $W_{M}^{s}(0)$ and $W_{M}^{u}(0)$ are oriented injectively immersed submanifolds of $\mathbf{R}^{2}$ with tangent space at 0 equal to the $y$-axis and $x$-axis respectively. Actually, by a theorem of Moser [M4, p. 674] there is a rectangle $R=\left\{\left(\begin{array}{l}\xi \\ \eta\end{array}\right) \in \mathbf{R}^{2}|| \xi\left|<2 d_{1},\right| \eta \mid<2 d_{2}\right\}$ and a symplectic diffeomorphism $P$ : $R \subseteq \mathbf{R}^{2} \rightarrow \mathbf{R}^{2}:\left(\begin{array}{l}\xi \\ \eta\end{array}\right) \rightarrow\left(\begin{array}{l}x \\ y\end{array}\right)$ such that

$$
N=P^{-1} M P: R \subseteq \mathbf{R}^{2} \rightarrow \mathbf{R}^{2}:\left(\begin{array}{l}
\xi \\
\eta
\end{array}\right) \rightarrow\left(\begin{array}{l}
\xi_{1} \\
\eta_{1}
\end{array}\right)=\left[\begin{array}{l}
(\lambda+P(\xi \eta)) \xi \\
(1 / \lambda+Q(\xi \eta)) \eta
\end{array}\right],
$$

where $P(0)=Q(0)=0$ and $1=(\lambda+P(\xi \eta))(1 / \lambda+Q(\xi \eta))$.

Thus $W_{N}^{s}(0) \cap R$ is the $\eta$-axis in $R$ while $W_{N}^{u}(0) \cap R$ is the $\xi$-axis in $R$.

Moreover for $(\xi, \eta) \in R \backslash\left\{\left(\begin{array}{l}\xi \\ \eta\end{array}\right) \in R \mid \xi=0\right.$ or $\left.\eta=0\right\}$ the following estimates hold $\left|\xi_{1}\right|>\lambda^{*}|\xi|$ and $\left|\eta_{1}\right|<1 / \lambda^{\prime}|\eta|$ for some $\lambda^{*}>1$ and $\lambda^{\prime}>1$, which describe the hyperbolic behaviour of $N$ in $R$.

A point $p \in \mathbf{R}^{2} \backslash\{0\}$ is a homoclinic point of $M$ if $p \in W^{s}(0) \cap W^{u}(0)$. The homoclinic point $p$ is of finite contact if for some nonnegative integer $k$ the $k$-jets of $W_{M}^{s}(0)$ and $W_{M}^{u}(0)$ at $p$ are equal but the $(k+1)$-jets at $p$ are unequal.

Now suppose that $M$ has a homoclinic point $p$ of finite contact. Since $p \in W_{M}^{s}(0)$ there is a positive integer $n$ such that $\tilde{p}=M^{n} p \in P(R)$. Since $W_{M}^{s}(0)$ and $W_{M}^{u}(0)$ have finite contact at $p$ and $M$ is a diffeomorphism, they have finite contact at $\tilde{p}$. Hence there is $\tilde{q} \in W_{M}^{u}(0) \cap P(R)$ with $\tilde{q} \notin W_{M}^{s}(0)$ $\cap P(R)$ such that the connected submanifold of $W_{M}^{u}(0)$ with boundary $\{\tilde{p}\} \cup\{\tilde{q}\}$ has the same orientation as $W_{M_{(}}^{u}(0)$.

Let $p_{1}=P^{-1} \tilde{p}$ and $q_{1}=P^{-1} \tilde{q}=\left(\begin{array}{c}\tilde{\xi} \\ )\end{array}\right)$. Then $\tilde{\xi} \neq 0$ because $q_{1} \notin$ 
$P^{-1}\left(W_{M}^{u}(0) \cap P(R)\right)=\mathscr{W}$ and $\tilde{\eta} \neq 0$ because $\mathscr{W}$ is injectively immersed.

Iterating the inequality $\left|\tilde{\xi}_{1}\right|>\lambda^{*}|\tilde{\xi}|$ where $\lambda^{*}>1$ shows that for every $c \in] 0, d_{1}\left[\right.$ there is a positive integer $l$, depending on $q_{1}$ and $c$, such that $\left|\tilde{\xi}_{l}\right|>c$, where $\left(\frac{\tilde{\xi}_{\tilde{\eta}}}{\eta_{1}}\right)=N^{l} q_{1}$. Thus $W$ intersects the line $\xi=c$ or $\xi=-c$.

Moreover, $\left|\tilde{\eta}_{l}\right|<2 d_{2}$, since $q_{1} \in R$, implies $|\tilde{\eta}|<2 d_{2}$. Thus the inequality $\left|\tilde{\eta}_{1}\right|<1 / \lambda^{\prime}|\tilde{\eta}|$ where $\lambda^{\prime}>1$ may be iterated. Repeating the above argument with $p_{k+1}=N^{k} p_{1}$ replacing $p_{1}$ shows that for an infinite sequence of distinct $c_{i}$ with $\left|c_{i}\right|<d_{1}$, W intersects the line segment $l_{c_{i}}=\left\{\left(\begin{array}{l}\xi \\ \eta\end{array}\right) \in \mathbf{R}^{2}\left|\xi=c_{i},\right| \eta \mid<\right.$ $\left.2 d_{2}\right\}$ in an infinite set $R_{c_{i}}$ (here we use the fact that $\vartheta$ is injectively immersed).

Now suppose that $M$ has an integral $H$ with $H(0)=h$, then $W_{M}^{u}(0) \subseteq$ $H^{-1}(h)$ for if $m \in W_{M}^{u}(0)$ then $h=H(0)=\lim _{n \rightarrow \infty} H\left(M^{-n} m\right)=H(m)$ because $H\left(M^{-n} m\right)=H(m)$.

Let $\tilde{H}=H \circ P: R \subseteq \mathbf{R}^{2} \rightarrow \mathbf{R}^{2}$, then $\tilde{H}$ is an integral for $N=P^{-1} M P$ in $R$ and thus $\mathscr{} \subseteq \tilde{H}^{-1}(h)$. Since $R_{c_{i}}$ has an accumulation point and $\tilde{H} \mid R_{c_{1}}=h$, $\tilde{H} \mid l_{c_{i}}=h$ by the identity theorem for real analytic functions. For every $\eta \in] 0,2 d_{2}[$ consider the function

$$
\tilde{H}_{\eta}:\left\{\xi|| \xi \mid<d_{1}\right\} \subseteq \mathbf{R} \rightarrow \mathbf{R}: \xi \rightarrow \tilde{H}(\xi, \eta) .
$$

Because $\tilde{H} \mid l_{c_{i}}=h, \tilde{H}_{\eta}\left(c_{i}\right)=h$ for all $\left.\eta \in\right] 0,2 d_{2}\left[\right.$ and therefore $\tilde{H}_{\eta}=h$ since $\tilde{H}_{\eta}$ is analytic and $\left\{c_{i}\right\}$ has an accumulation point.

Therefore $\tilde{H}=h$ on $R$ which implies $H$ is constant. This contradicts the assumption that $H$ is an integral of $M$. Therefore if $M$ is an integrable symplectic diffeomorphism of $\left(\mathrm{R}^{2}, d x \wedge d y\right)$ then $M$ has no homoclinic points of finite contact.

A recent result of Zehnder [Z] shows that a generic set of symplectic diffeomorphisms of $\left(\mathbf{R}^{2}, d x \wedge d y\right.$ ) have transversal (contact 0$)$ homoclinic points. Also numerical evidence seems to indicate that Moser's polynomial mapping in $\$ 2$ has a homoclinic point, although the author knows of no mathematical proof of this.

\section{REFERENCES}

[A] V. I. Arnol'd and A. Avez, Ergodic problems of classical mechanics, Gauthier-Villars, Paris, 1967; English transl., Benjamin, New York, 1968. MR 35 \#334; 38 \# 1233.

[AM] R. Abraham with J. Marsden, Foundations of mechanics, Benjamin, New York, 1967. MR 36 \#3527.

[B] R. B. Barrar, Isolated fixed points in celestial mechanics, J. Math. Anal. Appl. 36 (1971), 506-517. MR 44 \# 7049.

[C] R. Cushman, Analytic integrable area preserving diffeomorphisms of the closed 2-disc, Thesis, Princeton Univ., 1970.

[KN] S. Kobayashi and K. Nomizu, Foundations of differential geometry. I, Interscience, New York, 1970.

[M1] J. Moser, Nonexistence of integrals for canonical systems of differential equations, Comm. Pure Appl. Math. 8 (1955), 409-436. MR 18, 41. 
[M2] _ Stable and random motions in dynamical systems, Princeton Univ. Press, Princeton, N. J., 1973.

[M3] _ Lectures on Hamiltonian systems, Mem. Amer. Math. Soc. No. 81 (1968). MR 37 \#6060.

[M4] __ Analytic invariants of an area-preserving mapping near a hyperbolic fixed point, Comm. Pure Appl. Math. 9 (1956), 673-692. MR 19, 278.

[S] S. Smale, Differentiable dynamical systems, Bull. Amer. Math. Soc. 73 (1967), 747-817. MR 37 \# 3598; erratum, MR 39, p. 1593.

[Si] C. L. Siegel, On the integrals of canonical systems, Ann. of Math. (2) 42 (1941), 806-822. MR 3, 214.

[SM] C. Siegel and J. Moser, Lectures on celestial mechanics, Springer-Verlag, New York, 1971.

[Z] E. Zehnder, Homoclinic points near elliptic fixed points, Comm. Pure Appl. Math. 26 (1973), 131-182. MR 49 \#9873.

Mathematics Institute, RiJksUniversitert, Utrecht, THE NeTHERLANDS 